\title{
Interferometric Processing of ScanSAR Data Using Stripmap Processor: New Insights from Coregistration
}

\author{
Cunren Liang \\ Jet Propulsion Laboratory, California Institute of Technology, Pasadena, CA, USA \\ cunrenl@caltech.edu \\ Eric J. Fielding \\ Jet Propulsion Laboratory, California Institute of Technology, Pasadena, CA, USA \\ eric.j.fielding@jpl.nasa.gov
}

This is a post-print submitted to EarthArXiv before final IEEE editing and formatting. The paper is published as: C. Liang and E. J. Fielding, "Interferometric processing of ScanSAR data using stripmap processor: New insights from coregistration," IEEE Transactions on Geoscience and Remote Sensing, vol. 54, no. 7, pp. 4343-4354, Jul. 2016. DOI: 10.1109/TGRS.2016.2539962 


\title{
Interferometric Processing of ScanSAR Data Using Stripmap Processor: New Insights from Coregistration
}

\author{
Cunren Liang and Eric J. Fielding ${ }^{1}$
}

\begin{abstract}
Processing scanning synthetic aperture radar (ScanSAR) data using a stripmap processor, which is called full-aperture processing, has been the choice of many researchers. ScanSAR data are known to require very high azimuth coregistration precision which is usually achieved by a geometrical coregistration followed by a spectral diversity coregistration on the ScanSAR burst. However, for full-aperture processing, since individual bursts are no longer available for spectral diversity coregistration, the cross-correlation method in practice is still used to coregister ScanSAR data as stripmap data. We analyze the azimuth coregistration precision requirement of full-aperture processing, and find that its requirement can be significantly relaxed. This is confirmed by a number of experiments including simulations and real data experiments whose results are in good agreement with each other. An additional experiment on the cross-correlation method supports its use in full-aperture processing. Concluding from the experimental results, we further propose a simple method to evaluate the azimuth coregistration precision requirement for practical use. Finally, we present examples with ALOS-2 ScanSAR data.
\end{abstract}

Index Terms - scanning synthetic aperture radar (ScanSAR), coregistration, fullaperture, interferometry, ALOS-2.

\section{Introduction}

Wide swath imaging has been a developing direction of current spaceborne synthetic aperture radar (SAR) systems. Typical wide swath modes include terrain observation by progressive scans (TOPS) [1] mode and scanning synthetic aperture radar (ScanSAR) [2], [3] mode. A new wide-swath mode called SweepSAR [4] is planned for the National Aeronautics and Space Administration (NASA)-Indian Space Research Organisation (ISRO) SAR (NISAR) mission under development. Since the flight of NASA Shuttle Imaging Radar mission C (SIR-C) [5] in 1994, ScanSAR has been widely used in various spaceborne SAR missions, including Radarsat-1, Envisat, ALOS, COSMO-SkyMed, TerraSAR-X, and Radarsat-2. SAR interferometry (InSAR) has been benefitting from this wide swath mode to retrieve large-scale geophysical signals. This is the case especially with the recent 2014 launch of the Japanese Aerospace Exploration Agency (JAXA) Advanced Land Observation Satellite 2 (ALOS-2) mission [6] that is acquiring L-band ScanSAR data with controlled burst synchronization and small baselines, which is of great interest to the geophysical community.

Burst-by-burst and full-aperture approaches can be used to process ScanSAR InSAR data [7], [8]. Although the first approach is more standard, the latter one has been used by many researchers [9]-[11] because the overall spectral and geometrical

\footnotetext{
${ }^{1}$ The authors are with the Jet Propulsion Laboratory, California Institute of Technology, Pasadena, CA 91109 USA.

Copyright 2015 California Institute of Technology. U.S. Government sponsorship acknowledged.
} 
properties of the data processed in this way are similar to those of stripmap data. This consistency allows easy adaptation of a traditional stripmap InSAR processor to process ScanSAR data, except that the removal of azimuth non-overlap spectrum becomes very complicated for this approach. In particular, JAXA distributes this kind of phase-preserved full-aperture products for ALOS-2 ScanSAR, which have already been used to measure the large-scale deformation caused by the widely studied $\mathrm{Mw}$ 7.8 Gorkha Earthquake in Nepal that happened on April 25, 2015 [12], [13]. The resulting ALOS-2 ScanSAR interferograms are probably the most valuable interferograms for this large event, as they completely cover the large deformation field with good coherence despite the great topographic relief and significant vegetation cover in this area, which also shows the great potential of wide-swath Lband InSAR.

Burst mode interferometry usually requires very high azimuth coregistration precision. The commonly accepted coregistration strategy is composed of two steps. First, offsets of the InSAR pair are calculated using the geometrical information and a digital elevation model (DEM) [14]. Then the residual offsets are precisely estimated by the spectral diversity method [15], which is performed on the individual bursts. However, when the data are processed using a full-aperture approach, individual bursts are no longer available. Instead, the traditional cross-correlation coregistration is still used to coregister ScanSAR data. While successful applications of such fullaperture processing approach have been contributing to the modeling of earthquakes, it is not yet clear what is the azimuth coregistration requirement for the full-aperture approach and whether the cross-correlation method can meet this need. In the following sections, we analyze the azimuth coregistration requirement of full-aperture processing. We then carry out a series of experiments to confirm our findings and to consider different cases. Furthermore, an experiment is also designed to check the performance of cross-correlation method. Finally, we present a case study of ALOS2 using an azimuth coregistration precision requirement evaluation method proposed by us.

\section{Coregistration Requirement of Full-Aperture Approach}

\section{A. The Number of Looks of ScanSAR System}

We start our discussion with an accurate definition of the number of looks of a ScanSAR system, which is sometimes not very accurately defined. The discussion is limited to azimuth direction.

Suppose that, the full aperture length to be processed is $T_{A}$, burst length is $T_{B}$, and burst cycle is $T_{C}$, then the number of looks of the ScanSAR system can be defined as

$$
N_{L}=\frac{T_{A}-T_{B}}{T_{C}}
$$

The reason why there is a $T_{B}$ in the numerator is clearly shown in Fig. 1. Suppose the number of the subswaths of the ScanSAR system is $N_{S}$, then

$$
T_{C}=N_{S} T_{B} .
$$

Considering equation (1) and (2), $T_{B}$ can be given by

$$
T_{B}=\frac{T_{A}}{N_{L} N_{S}+1}
$$

B. ScanSAR Signal in Full-Aperture Processing 
If ScanSAR data are focused burst by burst, after focusing the azimuth signal of a point target illuminated by a burst can be written as [16]

$$
s_{f}(\eta)=T_{B} \operatorname{sinc}\left(K_{a} T_{B} \eta\right) \cdot \exp \left\{j \pi K_{a} \eta\left(\eta-2 \eta_{c}\right)\right\}
$$

where $\eta$ is the azimuth time, $\eta_{c}$ is the burst center for the point target, and $K_{a}\left(K_{a}>\right.$ 0 ) is the azimuth FM rate. We have ignored the range of closest approach, the antenna pattern, the weighting applied in the focusing and the complex constant modeling the backscattering characteristic. If the data are focused using the full-aperture approach, the focusing result is effectively a superposition of $N_{L}$ single burst signals focused burst by burst, and thus can be given by

$$
\begin{aligned}
s_{m f}(\eta) & =\sum_{l=1}^{N_{L}} s_{f}^{l}(\eta) \\
& =\sum_{l=1}^{N_{L}} T_{B} \operatorname{sinc}\left(K_{a} T_{B} \eta\right) \cdot \exp \left\{j \pi K_{a} \eta\left(\eta-2 \eta_{c}^{l}\right)\right\} .
\end{aligned}
$$

Although the signals of different bursts are superimposed before complex conjugate multiplication of the master and slave images in full-aperture processing, it can be proved that eventually (after low-pass filtering) we can still get an interferogram that is equivalent to

$$
i(\eta)=\sum_{l=1}^{N_{L}}\left\{s_{f, m}^{l}(\eta) \cdot\left[s_{f, s}^{l}(\eta)\right]^{*}\right\}
$$

where $m$ and $s$ denote master and slave, respectively. In equation (6), the superposition of the signals of different looks happens after the complex conjugate multiplication. Despite of the complex signal properties in full-aperture processing, we find this can provide an important way to analyze the phase error of the resulting interferogram caused by misregistration.

\section{Center Frequency of ScanSAR Signal in Full-Aperture Processing}

We first take a 3-look 3-subswath ScanSAR system as an example to show the center frequency of the ScanSAR signal in full-aperture processing. This is shown in Fig. 1 (b). For simplification, the Doppler centroid frequency is zero. In this case, equation (5) can be written as:

$$
\begin{aligned}
s_{m f}(\eta)= & \sum_{l=1}^{3} T_{B} \operatorname{sinc}\left(K_{a} T_{B} \eta\right) \cdot \exp \left\{j \pi K_{a} \eta\left(\eta-2 \eta_{c}^{l}\right)\right\} \\
= & T_{B} \operatorname{sinc}\left(K_{a} T_{B} \eta\right) \cdot\left[\exp \left\{j \pi K_{a} \eta\left(\eta-2 \eta_{c}^{1}\right)\right\}\right. \\
\quad & \left.\quad \exp \left\{j \pi K_{a} \eta\left(\eta-2 \eta_{c}^{2}\right)\right\}+\exp \left\{j \pi K_{a} \eta\left(\eta-2 \eta_{c}^{3}\right)\right\}\right] \\
= & T_{B} \operatorname{sinc}\left(K_{a} T_{B} \eta\right) \cdot \exp \left\{j \pi K_{a} \eta^{2}\right\} \\
& \cdot\left(\exp \left\{-j 2 \pi K_{a} \eta \eta_{c}^{1}\right\}+\exp \left\{-j 2 \pi K_{a} \eta \eta_{c}^{2}\right\}\right. \\
& \left.+\exp \left\{-j 2 \pi K_{a} \eta \eta_{c}^{3}\right\}\right)
\end{aligned}
$$

where 1, 2 and 3 denote the three consecutive bursts, respectively.

In Fig. 1(b), without considering the targets that are not fully illuminated by the burst and according to equation (3), the frequency duration of a burst in time domain is 


$$
T_{A}-T_{B}=9 T_{B} .
$$

which is also 3 times (looks) the duration of a burst cycle that is composed of 3 burst durations from 3 subswaths. That is, the frequency duration of a burst can be seen as being equally divided by 9 .

In equation (7), if we take burst 2 as reference, the time lags of burst 1 and burst 3 are $-3 T_{B}$ and $3 T_{B}$, respectively. Considering this, the last phase term in equation (7), which determines the center frequency, can be written as

$$
\begin{gathered}
p(\eta)=\exp \left\{-j 2 \pi K_{a} \eta\left(\eta_{c}^{2}-3 T_{B}\right)\right\}+\exp \left\{-j 2 \pi K_{a} \eta \eta_{c}^{2}\right\} \\
+\exp \left\{-j 2 \pi K_{a} \eta\left(\eta_{c}^{2}+3 T_{B}\right)\right\} \\
=\exp \left\{-j 2 \pi K_{a} \eta_{c}^{2} \eta\right\} \cdot\left[1+2 \cos \left(6 \pi K_{a} T_{B} \eta\right)\right]
\end{gathered}
$$

Note that we have been using relative time (variable $\eta$ ) to analyze the signals, where the zero time $(\eta=0)$ corresponds to the zero Doppler frequency of the point target under discussion. If we switch to absolute time and define the center of burst 2 as zero time, the center frequency of equation (9) is valid within

$$
\left[-\frac{3}{2} T_{B}, \frac{3}{2} T_{B}\right]
$$

This center frequency is denoted by the bold line in Fig. 1(b). Note that the bursts are the same, so this center frequency representation is repeated by each burst. As a result, there is a series of repeated bold lines in Fig. 1(b). On the other hand, the center frequency of burst 2 (or each burst) is valid within

$$
\left[-\frac{9}{2} T_{B}, \frac{9}{2} T_{B}\right]
$$

Therefore, the maximum center frequency of full-aperture signal is

$$
f_{\text {max,full aperture }}=\frac{f_{\text {max,single burst }}}{3} \text {. }
$$

where $f_{\text {max,single burst }}$ is the maximum center frequency of single burst.

\section{Phase Error Caused by Coregistration Error in Full-Aperture Processing}

According to equation (6), we can calculate the phase error of the resulting interferogram caused by misregistration. For each look, the phase error near the magnitude peak is dominated by the linear phase term in equation (4), while the influence of the quadratic phase term is usually ignored. Suppose that the coregistration error is $\Delta \eta$, the interferogram with the phase error can be written as

$$
\begin{aligned}
i(\eta)= & \exp \left\{j \Delta \theta^{1}\right\}+\exp \left\{j \Delta \theta^{2}\right\}+\exp \left\{j \Delta \theta^{3}\right\} \\
= & \exp \left\{-j 2 \pi K_{a} \eta_{c}^{1} \Delta \eta\right\}+\exp \left\{-j 2 \pi K_{a} \eta_{c}^{2} \Delta \eta\right\} \\
& \quad+\exp \left\{-j 2 \pi K_{a} \eta_{c}^{3} \Delta \eta\right\} \\
= & \exp \left\{-j 2 \pi K_{a}\left(\eta_{c}^{2}-3 T_{B}\right) \Delta \eta\right\}+\exp \left\{-j 2 \pi K_{a} \eta_{c}^{2} \Delta \eta\right\} \\
\quad & \quad+\exp \left\{-j 2 \pi K_{a}\left(\eta_{c}^{2}+3 T_{B}\right) \Delta \eta\right\} \\
= & \exp \left\{-j 2 \pi K_{a} \eta_{c}^{2} \Delta \eta\right\} \cdot\left[1+2 \cos \left(6 \pi K_{a} T_{B} \Delta \eta\right)\right]
\end{aligned}
$$

which follows the same rule as we have noted in the center frequency analysis. Here we have also used burst 2 as reference and ignored the magnitude. In equation (13), $\eta_{c}^{2}$ is also limited to the range expressed in equation (10). Therefore, the center frequency as shown in Fig. 1 can also be used in the analysis of the phase error caused. 
As a result, for the same coregistration error, the maximum phase error caused is only one third of that of single burst signal.

\section{E. Other Numbers of Looks}

Following similar analysis, we can calculate the phase errors caused by coregistration errors for other numbers of looks that may be used in practice. For $N_{L}=2$ and the same coregistration error, the maximum phase error is one half of that for single burst signal, which is shown in Fig. 1(a). For $N_{L}=4$ and the same coregistration error, the maximum phase error is one fourth of that for single burst signal, which is shown in Fig. 1(c). In these cases with an even number of looks, the zero center frequency of the full-aperture signal is now at the center of the burst gap, while in the previous case of 3 looks, the zero center frequency is at the center of the burst.

By summarizing these results, we can conclude that the maximum center frequency of the full-aperture signal is

$$
f_{\text {max }, \text { full aperture }}=\frac{f_{\text {max }, \text { single burst }}}{N_{L}}=\frac{\frac{N_{L} N_{S} T_{B}}{2} K_{a}}{N_{L}}=\frac{N_{S} T_{B}}{2} K_{a} .
$$

This is because the time lag between two consecutive bursts is $T_{C}=N_{S} T_{B}$ and the whole burst frequency duration is divided by $N_{L}$. Each divided part can be a burst cycle $T_{C}$, only within which the superimposition of the burst signals of different looks can happen. For odd and even number of looks, we have already seen the difference of the locations of zero center frequencies. This is because the whole burst frequency duration is divided by $N_{L}$ which is odd for odd number of looks, and even for even number of looks. When it is divided by an odd $N_{L}$, the center of the resulting burst cycle is exactly the center of the whole burst duration. On the other hand, when it is divided by an even $N_{L}$, the center of the whole burst duration is at the boundary of two resulting burst cycles. For both cases, the zero center frequency is at the center of the resulting burst cycle. This is the center of burst for odd $N_{L}$, and the center of burst gap for even $N_{L}$.

Until now, only the case of integer number of looks has been discussed. When the number of looks is not an integer, the problem is complicated. Too many discussions into the details are not meaningful to our purpose. Therefore, we only present an example of 2.5 looks in Fig. 1(d). In this case, the maximum phase error is one fifth of that of single burst signal.

Furthermore, practical cases are far more complicated. There are many factors that need to be considered. In the following sections, we will discuss the possible cases in the simulation and real data experiments.

\section{Simulation Experiments}

\section{A. Point Target Analysis}

We first show the significant phase error reduction of full-aperture processing by presenting a point target analysis example.

SAR systematic parameters from Envisat ASAR IS2 are used throughout the paper. Detailed parameters for this point target analysis example are shown in Table I. This is the same case shown in Fig. 1(b). The whole interferometric processes of both fullaperture processing and burst-by-burst processing are simulated. The coregistration error is $0.51 / \mathrm{PRF}$ for both cases. For the same coregistration error, the magnitude of 
the phase error varies with the azimuth location of the point target. To compare the results of the two cases, the point target with phase error of $1 / 3$ maximum phase error (Note that the maximum phase errors of the two cases are different. Here the maximum phase error refers to the maximum phase error of each case.) is chosen for each case.

The simulation results are shown in Fig. 2. Without phase error, the phase of the interferogram should be zero. As we can see from this figure, the phase error of the interferogram processed by full-aperture approach is much smaller than the phase error of single burst interferogram.

By inspecting the phase error of the interferogram, we can also see that there is a phase slope for each case. For the single burst interferogram, this is caused by the quadratic phase term in equation (4). According to equation (6), a similar phase slope exists in the full-aperture interferogram. Since the phase with peak magnitude dominates the interferometric phase, this is not considered any longer.

\section{B. Simulation Results of Different Numbers of Looks}

The same simulation process is carried out for all the cases plotted in Fig. 1. For each case, we do the simulation for point targets with different azimuth locations. The azimuth range is $3 N_{L} T_{C}$. The simulation is started with the point target that is exactly located at the center of a burst. We still use a coregistration error of $0.51 / \mathrm{PRF}$. In this way, we can observe how phase error varies with the azimuth location of the point target and most importantly, how big the maximum phase error is.

The simulation results are presented in Fig. 3. We take 3 looks shown in Fig. 3(b) as an example to explain the simulation results. Theoretically, the maximum phase error of single burst signal is [15]

$$
E_{\text {max }}=2 \pi\left(\frac{N_{L} N_{S} T_{B}}{2} K_{a}\right) \Delta \eta
$$

According to the parameters shown in Table I, this results in a maximum phase error of $1.02 \mathrm{rad}$ which is exactly the maximum phase error shown in Fig. 3(b). According to the previous analysis, the maximum phase error of full-aperture processing should be $E_{\max } / 3$. However, the simulated maximum phase error is only $0.11 \mathrm{rad}$ which is only $E_{\max } / 9$. This is because of the signal that is shorter than one burst, which has been ignored in the discussion. While this signal is usually not focused in burst-byburst approach due to its lower resolution, it is focused in the full-aperture approach. In Fig. 1(b), the time span of the bold line is $3 T_{B}$, which can be equally divided by 3 as shown in the figure. Only the middle part with a length of $T_{B}$ is not superimposed by this kind of signal. This makes the maximum phase error of full-aperture processing only $1 / 9$ of that of single burst signal. In particular, the maximum phase error happens at the burst edge, while zero phase error happens at the burst center, which is in well agreement with Fig. 1(b). Within the time span of the middle part of the bold line, the phase error increases linearly as the case of single burst. For the other two parts, the phase error decreases approximately linearly due to the influence of the signal shorter than $T_{B}$. The length of this signal varies with the azimuth location of the target.

From this simulation, we can see that the maximum phase error of full-aperture processing is reduced due to the reason discussed in the theoretical analysis, and 
further reduced due to the signal on burst edge that is shorter than $T_{B}$. This makes it significantly smaller than the maximum phase error of single burst.

Similarly, as shown in Fig. 3, the maximum phase error of full-aperture processing of 2-look case is $0.16 \mathrm{rad}$, which is $1 / 6$ of that of single burst. For 4 -look case, the maximum phase error of full-aperture processing is $0.09 \mathrm{rad}$, which is $1 / 12$ of that of single burst. For 2.5-look case, the maximum phase error of full-aperture processing is $0.20 \mathrm{rad}$, which is $1 / 5$ of that of single burst.

\section{Extensive Simulations}

In the previous simulations, we have fixed the number of subswaths and only selected four cases for the number of looks. In this subsection, we do simulation for a subswath range of $[2,6]$ and a look range of $[2,4]$. This should have included most of the practical cases. Misregistration of $0.51 / \mathrm{PRF}$ is still used in the simulation.

We also use a non-integer number of subswaths, to take into account the fact that, in practice, some pulses of a burst are lost due to the switch of the antenna elevation angle. In addition, the switch itself also takes time. These all make the burst of a subswath shorter. If the burst cycle is fixed, this can be equivalent to an increase of the number of subswaths.

The maximum phase error of full-aperture processing is shown in Fig. 4(a). As we can see from this figure, the maximum phase error increases as the number of subswath $N_{S}$ increases. Two factors have contributed to this increase. First, as shown by equation (3), $T_{B}$ decreases as $N_{S}$ increases. Signal shorter than $T_{B}$ only exists in the two time spans at the two ends of the bold line shown in Fig. 1. The duration of each time span is $T_{B}$ that decreases. Second, according to equation (2) and (3), the burst cycle can be given by

which increases as $N_{S}$ increases.

$$
T_{C}=\frac{N_{S} T_{A}}{N_{L} N_{S}+1}
$$

In general, the maximum phase error decreases as the number of looks $N_{L}$ increases because the whole burst duration $T_{A}-T_{B}$ is divided by a larger number. When the number of looks is not an integer, the variation of the maximum phase error is different, which is too trivial to discuss. In this case, the readers can refer to the 2.5look example.

On the other hand, the maximum phase error shown in Fig. 4(b) does not change much with the number of subswaths and the number of looks, which is reasonable. To further compare the two maximum phase errors, we compute their ratio which is presented in Fig. 4(c). A profile of Fig. 4(c) is presented in Fig. 4(d).

The simulation result can be a reference when we choose the total azimuth bandwidth to be processed in the azimuth focusing to minimize the maximum phase error.

Another problem that may be of interest is how phase error varies with coregistration error. From equation (13), we can see that their relationship is linear. This is confirmed by the simulation result shown in Fig. 5.

\section{Simulation and Real Data Experiments Considering Different Cases}


In practice, several factors including weighting on the azimuth spectrum, zero Doppler centroid frequency, and burst synchronization may affect the interferometric phase error caused by misregistration. In this section, we study their effects through both simulation and real data experiments. In the mean time, this is also a crossvalidation of the simulation and real data experimental results.

For these experiments, we use the mostly analyzed 3-subswath and 3-look case. Burst mode data are also from Envisat ASAR IS2. Coregistration error of 0.5 1/PRF is still used.

For real data experiments, master and slave data are from the same acquisition. This reduces the phase noise of the interferogram and directly shows the phase error in the interferogram. To compare the simulation and real data results, we first use a mean value filter to filter the interferogram in the azimuth direction and then average all the samples in the range direction. In this way, a column average of the interferogram is generated. Its phase is compared with simulation result. An interferogram that is looked in both range and azimuth directions in a regular way is also generated.

\section{A. The Simplest Case}

The experimental results of the simplest case are presented in Fig. 6. In this case, the azimuth spectrum is not weighted, and even the original antenna pattern weighting is removed in the processing. The Doppler centroid frequency is $0 \mathrm{~Hz}$. The burst synchronization is $100 \%$. This is exactly the case of our simulations in the previous section. Fig. 6(a) shows that the simulation and real data results are in good agreement with each other. The interferogram in Fig. 6(b) shows a wave-like phase error. This is a further validation of the discussions in the previous sections.

\section{B. The Effect of Weighting on Azimuth Spectrum}

Weighting is usually applied in the stripmap SAR signal processing [16]. For ScanSAR, this is also related to the suppressing of azimuth scalloping. Two experiments are carried out to study the effect of weighting on interferometric phase error caused by misregistration. All conditions are kept the same as the simplest case for both experiments, except the weighting on azimuth spectrum.

In the first experiment, the azimuth spectrum is weighted by the original azimuth antenna pattern of the Envisat ASAR IS2 data. The results are shown in Fig. 7. In this case, the maximum phase error becomes $0.09 \mathrm{rad}$ that is smaller than that of the case without any weighting. If we further apply a Kaiser window with a beta value of 2.5 to the azimuth spectrum, the maximum phase error is 0.04 rad which is significantly smaller. This result is shown in Fig. 8. As we can see from Fig. 8(b), the phase error is not very evident in the interferogram in this case.

By comparing these results with result without weighting, we can see that the phase error slope in the azimuth direction is reduced. This is the consequence of applying the weighting to the azimuth spectrum. The weighting has suppressed the high frequency components of the spectrum, making the increase of the phase error in the azimuth direction much slower.

To further study the reduction of phase error due to the weighting of azimuth spectrum, we change the beta value of the Kaiser window. We then only apply this weighting to the azimuth spectrum (no azimuth antenna pattern applied), and calculate the maximum phase error for each beta value. The simulation result is 
shown in Fig. 9. The result shows that the maximum phase error drops quickly as the weighting becomes stronger.

\section{The Effect of Doppler Centroid Frequency}

If Doppler centroid frequency is not zero, the whole single burst spectrum of the target will be moved by Doppler centroid frequency. This should be the same case for full-aperture signal. Therefore, the overall phase error caused should also include the part that is caused by non-zero Doppler centroid frequency.

We carry out an experiment to verify this. This experiment is the same as the one in the simplest case with only Doppler centroid frequency changed to $200 \mathrm{~Hz}$. The experimental results are shown in Fig. 10. As expected, the amplitude of the phase error wave is $0.11 \mathrm{rad}$, the same as the simplest case. The whole phase error wave is moved by $0.38 \mathrm{rad}$ which is exactly the phase error due to the $200 \mathrm{~Hz}$ Doppler centroid frequency that can be calculated by $2 \pi f_{D C} \Delta \eta$, where $f_{D C}=200 \mathrm{~Hz}$, and $\Delta \eta=1 / 2 \mathrm{PRF}$.

To further verify this, we change the value of the Doppler centroid frequency to do the same simulation and calculate the maximum phase error caused. The result is shown in Fig. 11. As expected, the maximum interferometric phase error changes linearly with Doppler centroid frequency.

\section{The Effect of Burst Synchronization}

ScanSAR interferometry requires burst synchronization so that there is common azimuth spectrum between the master and slave data. Current spaceborne sensors are usually under good control to achieve very high burst synchronization. Exactly quantifying the effect of burst synchronization on the phase error caused by misregistration is difficult. We do an experiment to study this.

In this real data experiment, the number of unsynchronized echoes is 30 , corresponding to a burst synchronization of $67 \%$. To suppress phase noise, we use a longer mean value filter to filter the interferogram in the azimuth direction. All other conditions are kept the same as the simplest case. For the simulation experiment, it's difficult to fully simulate this case. Instead, simulation result of the simplest case is used to compare with real data result. The results are shown in Fig. 12. Compared with the results in Fig. 6, the phase error wave is moved left by about 15 1/PRF. This is reasonable, since the shift is half of the number of unsynchronized echoes. Actually, the simulated result is also moved in the same way in order to compare with real data result. The maximum phase error is slightly larger than that of the simplest case. Overall, the phase error is not significantly different in this case.

\section{A Realistic Case}

In the experiments of the previous sections, we have specifically set the parameters to study the phase error caused by misregistration. In this section, we use two acquisitions to form the InSAR pair and consider many factors to present a more realistic case. On the other hand, our discussion has been focused on the reduction of misregistration error in the full-aperture processing. Although it is significantly smaller than that of single burst case, we are still interested in if the traditional crosscorrelation coregistration method that is widely used in the stripmap InSAR processor can also fulfill the coregistration requirement of full-aperture ScanSAR processing. This also needs further experiments. 
For the ScanSAR data and the full-aperture processing, compared with the simplest case in Section 10, the differences are as follows. We use data acquired on two dates for the InSAR pair. Only the original azimuth antenna pattern is applied to the azimuth spectrum. We don't choose to apply additional weighting because we still want to check the phase error wave; otherwise, the phase error may be too small to check. The Doppler centroid frequency is $200 \mathrm{~Hz}$. The number of unsynchronized echoes is 30 (67\% burst synchronization). This should be adequate to be considered as a realistic case. This InSAR pair is processed using a regular stripmap InSAR processing flow. In particular, the cross-correlation method is used to coregister the pair.

To check the phase error caused by misregistration and the performance of crosscorrelation method in the full-aperture processing, the original Envisat ASAR IS2 data are also processed as stripmap data in the same way.

We first present the coregistration results as shown in Fig. 13, from the crosscorrelation program ampcor from ROI_pac [17]. In general, the stripmap InSAR coregistration results are slightly better than ScanSAR coregistration results. In Fig. 13(c) and (d), more offsets of ScanSAR coregistration are scattered in the plots. However, the number of these erroneous offsets is small compared with the overall number of offsets calculated. In addition, the widths of the center lines composed of dense offsets in the plots of ScanSAR coregistration results are a little bit larger. The increased decorrelation including the increased volume scattering decorrelation [18] contributes to the worse coregistration results of ScanSAR pair. The big difference is in the azimuth offsets, where there are three lines of dense numbers of offsets in Fig. 13(d). The two additional lines are caused by the "spikes" in the full-aperture processing results [19]. The distance of the lines is about $51 /$ PRF which is exactly the distance of the spikes shown in Fig. 2. The resolution of the spikes, that is the resolution of the stripmap signal, determines the coregistration precision [19] after removing the offsets forming the two additional lines. For practical data processing, special attention should be paid to this kind of offsets to remove them; otherwise, severe interferometric phase error may be caused.

To further compare azimuth coregistration results, we fit a polynomial to the azimuth offsets. The polynomial is then used to calculate an azimuth offset map. The results are shown in Fig. 14(a) and (b). The difference map of the two azimuth offset maps is shown in Fig. 14(c). As we can see from this figure, there is nearly no difference between the two azimuth offset maps. Note that the ScanSAR azimuth bandwidth is only about $1 / 14$ PRF. The difference map is further converted to phase error map according to the simulation result, which is shown in Fig. 14(d). As we can see from this figure, the phase error is very small and can be ignored.

The interferograms are presented in Fig. 15. The stripmap interferogram can be seen as the interferogram without phase error caused by misregistration. Therefore, to check the phase error of ScanSAR interferogram caused by misregistration, the differential interferogram of the two interferograms is generated and presented in Fig. 15(c). There is no evident phase error in this figure, apart from the phase noise. In particular, there is no wave-like phase error. This implies that the cross-correlation has met the needs of full-aperture processing.

To further validate the conclusions, we add a 0.5 1/PRF coregistration error to azimuth ScanSAR coregistration result. The resulting interferogram is presented in Fig. 16(b). Now the differential interferogram shown in Fig. 16(c) shows the wave- 
like phase error which cannot be seen in Fig. 16(b). It's clear that the mean value of the wave is not zero, which should be caused by the $200 \mathrm{~Hz}$ Doppler centroid frequency. As in the previous section, the differential interferograms of both cases are averaged in the range direction and plotted in Fig. 17. For the case without the 0.5 1/PRF coregistration error, there is no evident phase error as shown in Fig. 17(a). For the case with the $0.51 / \mathrm{PRF}$ coregistration error, the real data result is in good agreement with the equivalent simulation result as shown in Fig. 17(b), despite of the phase noise. This agreement is an effective validation of the previous conclusions.

\section{Application to ALOS-2}

Based on the experimental results of the previous sections, we introduce the following method to check the coregistration precision requirement of a specific full-aperture processing case. The same image processed by full-aperture approach is used for both master and slave images. The slave image is shifted by a small offset in the azimuth direction. An interferogram can be formed using the two images. The interferogram is then filtered by a mean value filter. Finally, all the range samples are averaged to generate a column of interferogram, the phase of which is the phase error caused by the small azimuth offset.

We use this method to check the coregistration precision requirement of the standard ALOS-2 full-aperture product. Some systematic parameters of ALOS-2 can be found in [6], [12]. Since early February 2015, the burst synchronization of ALOS-2 has been under good control. The high coherence interferograms processed by software packages such as GMTSAR [20] and ISCE [21], using its full-aperture products have significantly contributed to the research of the April 25, 2015 Nepal earthquake in the geophysical community.

The data used were acquired on May 3, 2015. There are 5 subswaths and two polarizations. The overall swath width is about $350 \mathrm{~km}$. We present the experimental results of $\mathrm{HH}$ polarization data of subswath 1 . The cross-correlation results of this data and the corresponding data acquired on April 05, 2015 are shown in Fig. 18. As expected, there are additional lines in the plot of azimuth offset measurement results, which should be caused by the spikes of the full-aperture product. In general, the coregistration results are good.

We then check the coregistration precision requirement only using the May 3, 2015 acquisition. The coregistration error is 1 1/PRF, where PRF equals to $2661.847 \mathrm{~Hz}$. The result is presented in Fig. 19. We first examine the azimuth spectrum of the product which is shown in Fig. 19(a) in a frequency range of [-PRF/2, PRF/2]. We can see that the entire azimuth spectrum was processed when the product was generated. Furthermore, the azimuth spectrum is weighted. The phase error and the interferogram are shown in Fig. 19(b) and (c), respectively. Obviously, the wave-like error is shifted upwards, which should be caused by the Doppler centroid frequency. The maximum phase error is about 0.15 rad. Suppose that azimuth coregistration using cross-correlation can achieve a precision of $0.11 / \mathrm{PRF}$, the resulting phase error should be only $0.015 \mathrm{rad}$ which is equivalent to a deformation error of $0.29 \mathrm{~mm}$.

\section{Conclusion}

In this paper, we have studied the coregistration precision requirement of full-aperture ScanSAR InSAR processing. The interferometric phase error caused by misregistration is significantly reduced due to the superimposition of signals from different bursts, the signals shorter than one burst on the burst edges, and the 
weighting on the azimuth spectrum. The phase error caused by misregistration increases with the number of subswaths, Doppler centroid frequency and misregistration.

On the other hand, due to the nature of the image processed by full-aperture approach, the traditional cross-correlation method still achieves very high azimuth coregistration precision despite of the low azimuth resolution of the data [19]. In general, the crosscorrelation method can be used in the full-aperture approach to coregister the InSAR data acquired by a multi-look ScanSAR system considering the reduction of the interferometric phase error and the high coregistration precision achieved, as long as the cross-correlation matches are culled carefully. It is known that the rationality of using a stripmap InSAR processor to process ScanSAR data is mainly because of the consistency of the overall spectral and geometrical properties. We show here that it also extends to coregistration.

Based on the experimental results, we introduce a simple method to evaluate the coregistration precision requirement of full-aperture processing. We apply this method to the ALOS-2 full-aperture ScanSAR product. The experimental results can be a reference for using a traditional stripmap InSAR processor to process this kind of product.

\section{Acknowledgment}

The Envisat ASAR data are provided by the European Space Agency (ESA). The ALOS-2 ScanSAR original data are copyright JAXA and provided under JAXA RA4 PI projects P1372002 and P1385002. This research was supported by the NASA Earth Surface and Interior focus area and performed at the Jet Propulsion Laboratory (JPL), California Institute of Technology. This research was also supported by an appointment to the NASA Postdoctoral Program at JPL.

\section{References}

[1] F. De Zan and A. Monti Guarnieri, "TOPSAR: Terrain observation by progressive scans," IEEE Trans. Geosci. Remote Sens., vol. 44, no. 9, pp. 2352-2360, Sep. 2006.

[2] K. Tomiyasu, "Conceptual performance of a satellite borne, wide swath synthetic aperture radar," IEEE Trans. Geosci. Remote Sens., vol. GE-19, no. 2, pp. 108$116,1981$.

[3] R. K. Moore, J. P. Claassen, and Y. H. Lin, "Scanning spaceborne synthetic aperture radar with integrated radiometer," IEEE Trans. Aerosp. Electron. Syst., vol. AES-17, no. 3, pp. 410-420, 1981.

[4] A. Freeman, G. Krieger, P. Rosen, M.Younis, W. T. K. Johnson, S. Huber, R. Jordan, and A. Moreira, "SweepSAR: Beam-forming on receive using a reflectorphased array feed combination for spaceborne SAR," in Proc. 2009 IEEE Radar Conference, Pasadena, CA, USA, May 4-8, 2009.

[5] B. L. Huneycutt, "Spaceborne imaging radar-C instrument," IEEE Trans. Geosci. Remote Sens., vol. 27, no. 2, pp. 164-169, Mar. 1989.

[6] Y. Kankaku, Y. Osawa, S. Suzuki, and T. Watanabe, "The overview of the L-band SAR onboard ALOS-2," in Proc. PIERS, Moscow, Russia, Aug. 18-21, 2009, pp. 735-738.

[7] A. Monti Guarnieri and C. Prati, "ScanSAR focusing and interferometry," IEEE Trans. Geosci. Remote Sens., vol. 34, no. 4, pp. 1029-1038, July 1996. 
[8] R. Bamler and M. Eineder, "ScanSAR processing using standard high precision SAR algorithms," IEEE Trans. Geosci. Remote Sens., vol. 34, no. 1, pp. 212-218, Jan. 1996.

[9] X. Tong, D. T. Sandwell, and Y. Fialko, "Coseismic slip model of the 2008 Wenchuan earthquake derived from joint inversion of interferometric synthetic aperture radar, GPS, and field data," J. Geophys. Res., vol. 115, no. B4, pp. B04314, Apr. 2010.

[10] E. J. Fielding, A. Sladen, Z. Li, J.-P. Avouac, R. Bürgmann, and I. Ryder, "Kinematic fault slip evolution source models of the 2008 M7.9 Wenchuan earthquake in China from SAR interferometry, GPS and teleseismic analysis and implications for Longmen Shan tectonics," Geophys. J. Int., vol. 194, no. 2, pp. 1138-1166, May 2013.

[11] C. Liang, Q. Zeng, J. Jia, J. Jiao, and X. Cui, "ScanSAR interferometric processing using existing standard InSAR software for measuring large scale land deformation," Comput. Geosci., vol. 51, pp. 439-448, Feb. 2013.

[12] E. O. Lindsey, R. Natsuaki, X. Xu, M. Shimada, M. Hashimoto, D. Melgar, and D. T. Sandwell, "Line of sight displacement from ALOS-2 interferometry: Mw 7.8 Gorkha earthquake and Mw 7.3 aftershock," Geophys. Res. Lett., doi:10.1002/2015GL065385.

[13] J. Galetzka, D. Melgar, J. F. Genrich, J. Geng, S. Owen, E. O. Lindsey, X. Xu, Y. Bock, J.-P. Avouac, L. B. Adhikari, B. N. Upreti, B. Pratt-Sitaula, T. N. Bhattarai, B. P. Sitaula, A. Moore, K. W. Hudnut, W. Szeliga11, J. Normandeau, M. Fend, M. Flouzat, L. Bollinger, P. Shrestha, B. Koirala, U. Gautam, M. Bhatterai, R. Gupta, T. Kandel, C. Timsina, S. N. Sapkota, S. Rajaure, and N. Maharjan, "Slip pulse and resonance of the Kathmandu basin during the 2015 Gorkha earthquake, Nepal," Science, Aug. 2015.

[14] E. Sansosti, P. Berardino, M. Manunta, F. Serafino, and G. Fornaro, "Geometrical SAR image registration," IEEE Trans. Geosci. Remote Sens., vol. 44, no. 10 , pp. 2861-2870, Oct. 2006.

[15] R. Scheiber and A. Moreira, "Coregistration of interferometric SAR images using spectral diversity," IEEE Trans. Geosci. Remote Sens., vol. 38, no. 5, pp. 2179-2191, Sep. 2000.

[16] I. G. Cumming and F. H. Wong, Digital Processing of Synthetic Aperture Radar Data: Algorithms and Implementation. Norwood, MA, USA: Artech House, 2005.

[17] P. A. Rosen, S. Hensley, G. Peltzer, and M. Simons, "Updated repeat orbit interferometry package released," Eos Trans. $A G U$, vol. 85, no. 53, pp. 47, Feb. 2004.

[18] A. Monti Guarnieri and F. Rocca, "Combination of low- and high-resolution SAR images for differential interferometry," IEEE Trans. Geosci. Remote Sens., vol. 37, no. 4, pp. 2035-2049, Jul 1999.

[19] J. Holzer and R. Bamler, "Burst-mode and ScanSAR interferometry," IEEE Trans. Geosci. Remote Sens., vol. 40, no. 9, pp. 1917-1934, Sep. 2002.

[20] D. Sandwell, R. Mellors, X. Tong, M. Wei, and P. Wessel, "Open radar interferometry software for mapping surface deformation," Eos Trans. AGU, vol. 92, no. 28, pp. 234-235, Jul. 2011.

[21] P. A. Rosen, E. Gurrola, G. F. Sacco, and H. Zebker, "The InSAR scientific computing environment," in Proc. EUSAR, Nuremberg, Germany, Apr. 23-26, 2012, pp. 730-733. 


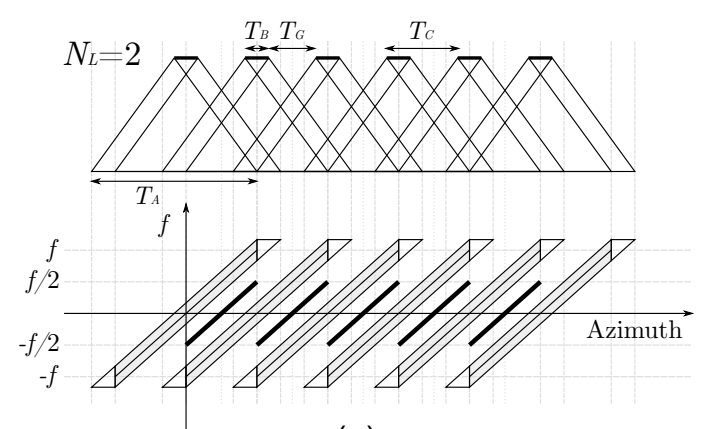

(a)
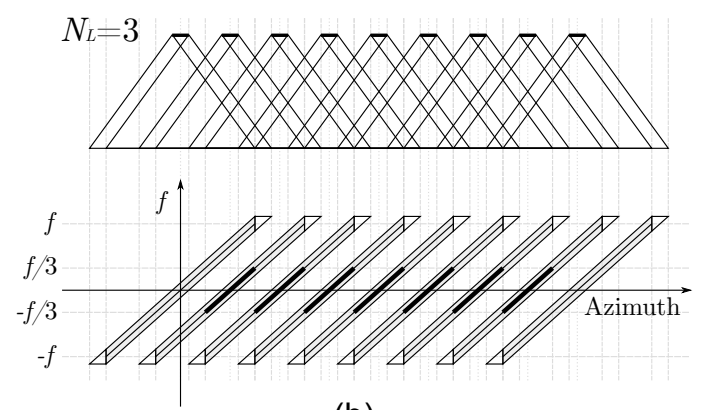

(b)

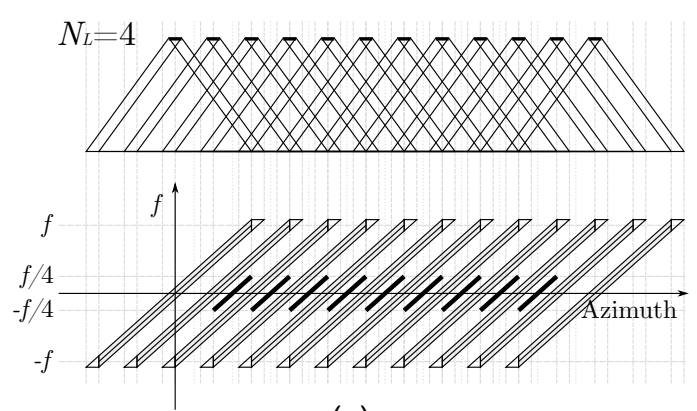

(c)

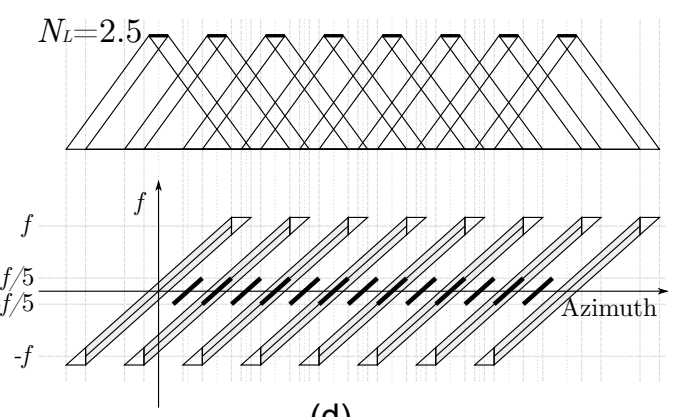

(d)

Fig. 1. Azimuth time-frequency plots of ScanSAR bursts. (a) 2 looks. (b) 3 looks. (c) 4 looks. (d) 2.5 looks. The number of subswath is 3 . Doppler centroid frequency is zero. $T_{B}$ is burst length, $T_{G}$ is burst gap length, and $T_{A}$ is full aperture length. The center lines of the grey strips indicate the center frequencies of the burst signals. The bold lines indicate the center frequencies of the sums of the burst signals. Signals on the burst edges shorter than the bursts are not considered in the sums. Vertical dotted lines indicate the zero frequencies of the sums of the burst signals. Vertical dashed lines indicate the frequency boundaries of the bursts. Horizontal dashed lines indicate the maximum center frequencies. 


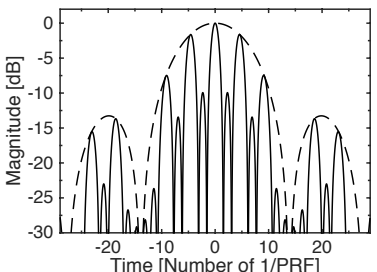

(a)

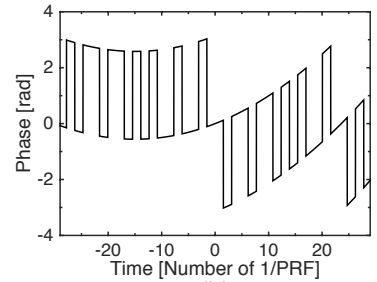

(b)

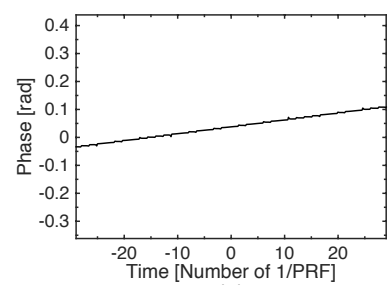

(c)

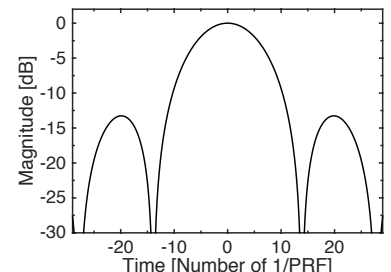

(d)

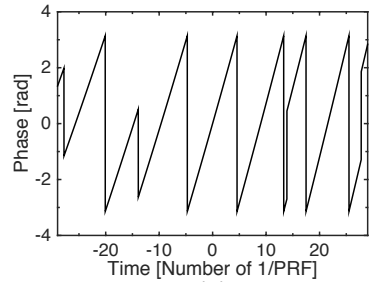

(e)

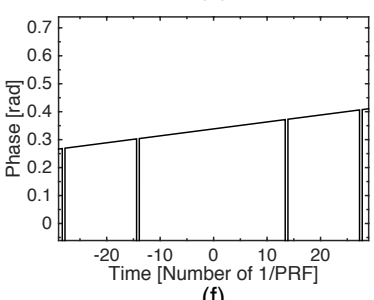

(f)

Fig. 2. A comparison of phase error caused by misregistration. The left and right columns are processed by full-aperture and burst-by-burst approaches, respectively. The first and second rows are the magnitude and phase of the point target. The third row is the phase of the interferogram with a coregistration error of $0.51 / \mathrm{PRF}$. Note that (c) is filtered by a mean value filter. The dashed line in (a) shows the envelope.
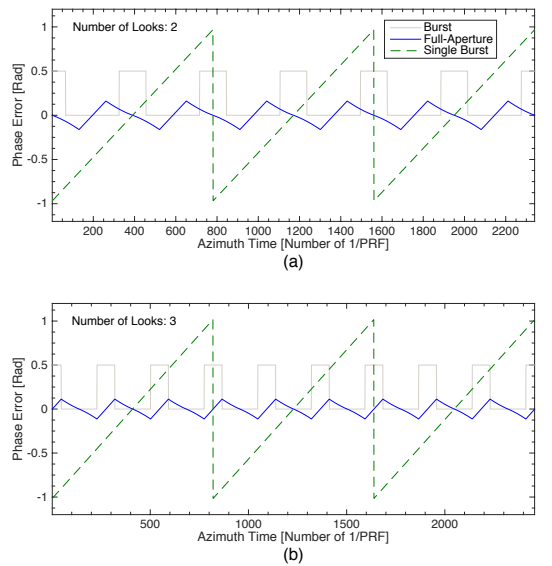
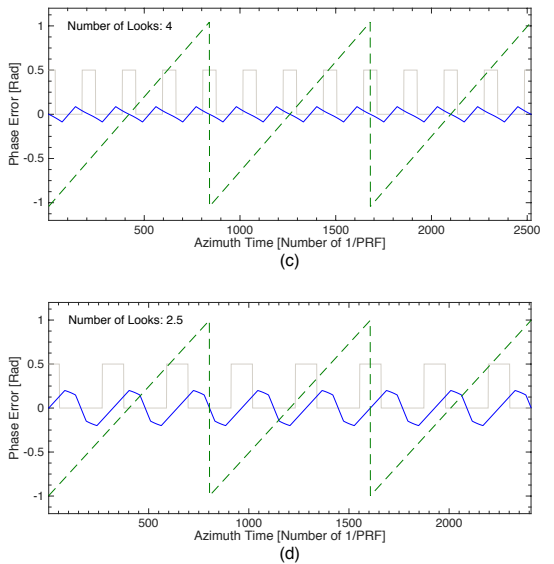

Fig. 3. Simulated interferometric phase error caused by a coregistration error of 0.5 1/PRF. (a) 2 looks. (b) 3 looks. (c) 4 looks. (d) 2.5 looks. Grey lines in the figures denote bursts and burst gaps. 

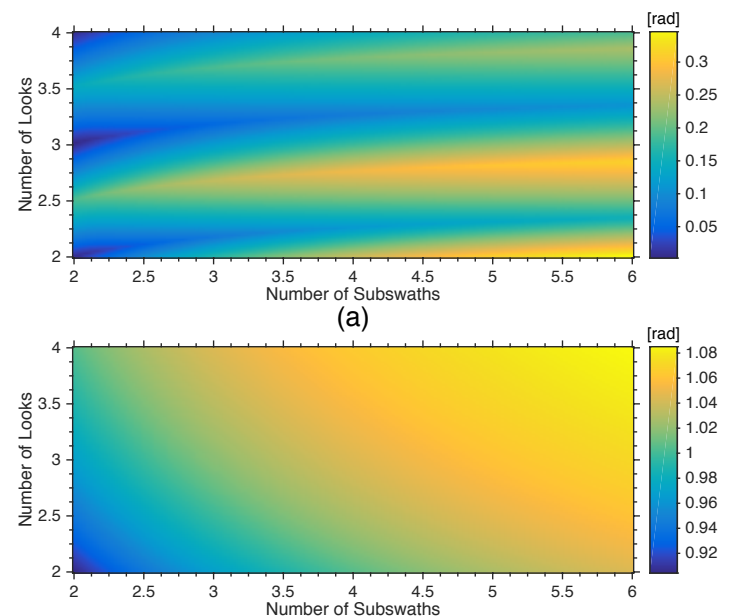

(b)

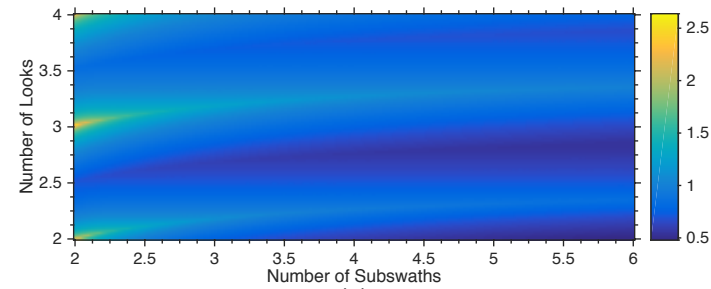

(c)

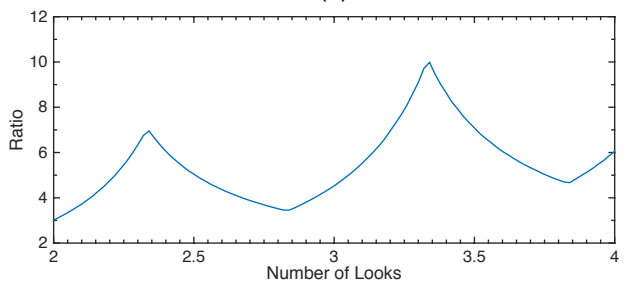

(d)

Fig. 4. (a) Maximum phase error as a function of the number of subswaths and the number of looks for full-aperture case. (b) Maximum phase error as a function of the number of subswaths and the number of looks for single burst case. (c) The ratio of (b) and (a) shown in $\log _{10}$. (d) Profile along last column of (c).

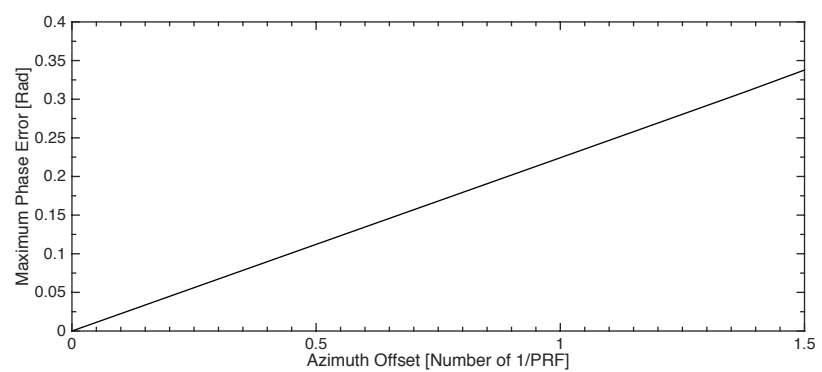

Fig. 5. The simulated relationship of interferometric phase error and coregistration error. 


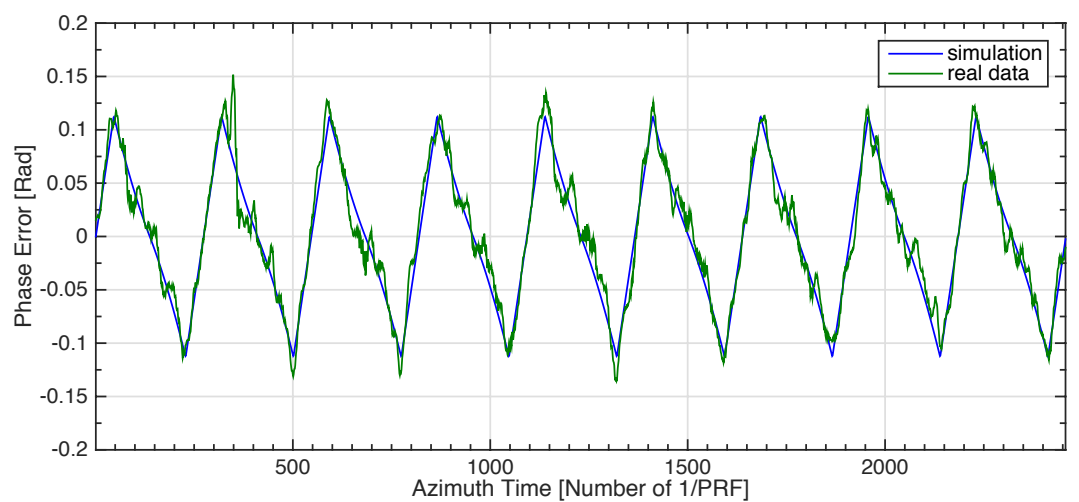

(a)

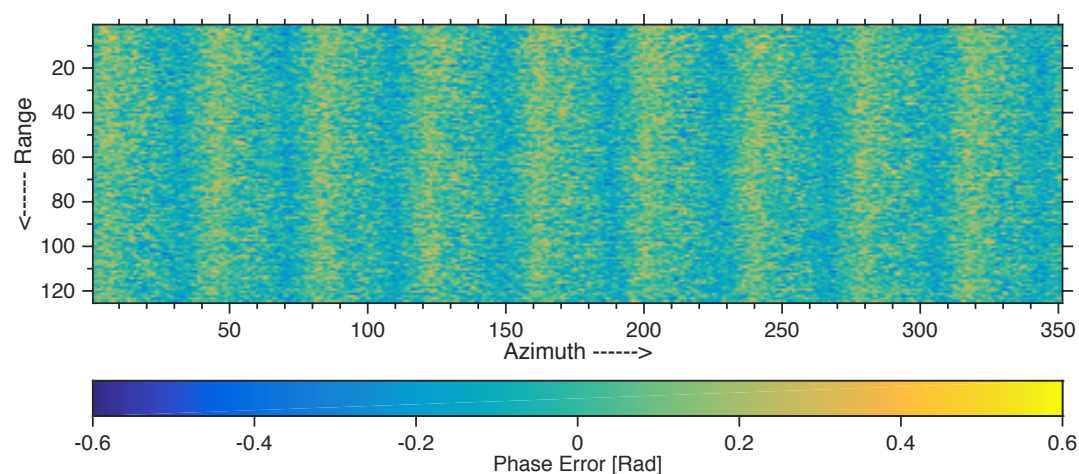

(b)

Fig. 6. Interferometric phase error caused by misregistration. No weighting of azimuth spectrum. Doppler centroid frequency is $0 \mathrm{~Hz}$. Burst synchronization is $100 \%$. (a) Comparison of simulation and real data results. (b) Interferogram of real data experiment. 


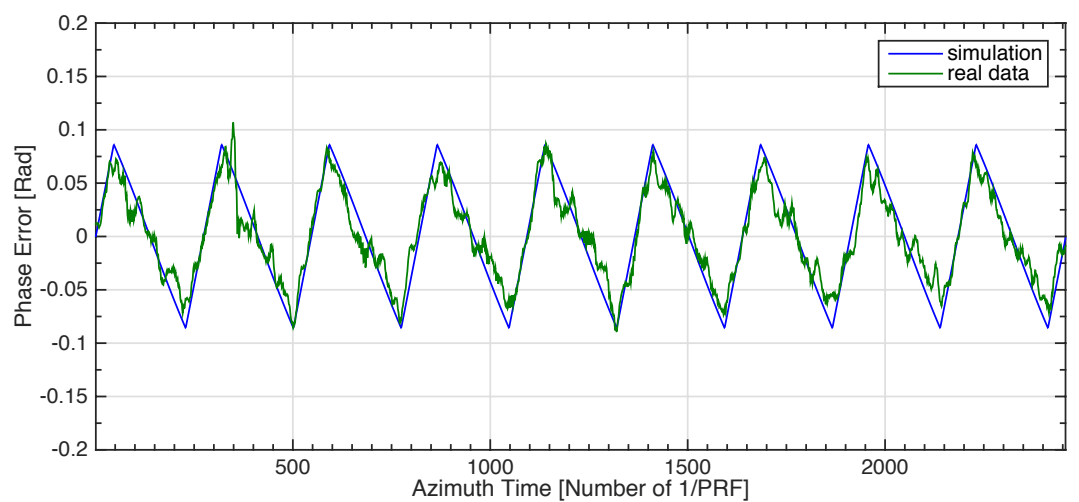

(a)

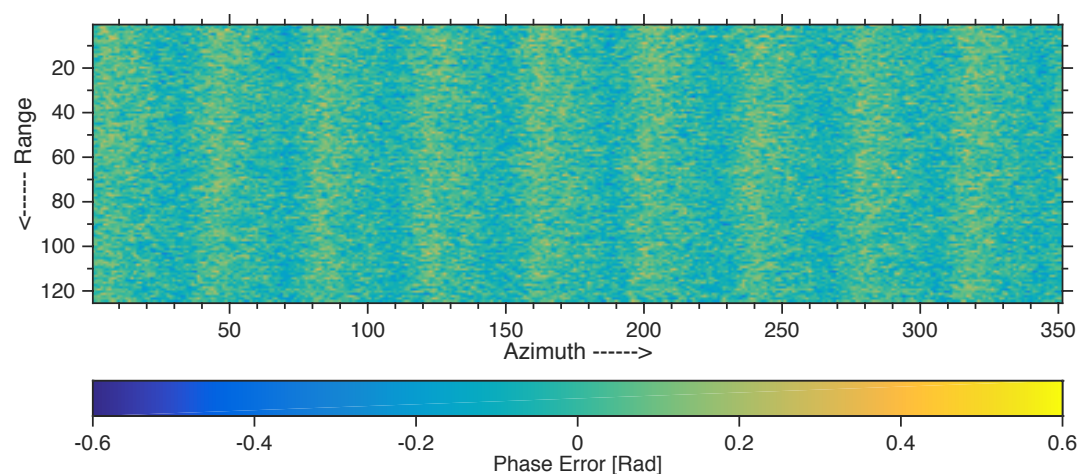

(b)

Fig. 7. Interferometric phase error caused by misregistration. Azimuth spectrum weighted by azimuth antenna pattern. Doppler centroid frequency is $0 \mathrm{~Hz}$. Burst synchronization is $100 \%$. (a) Comparison of simulation and real data results. (b) Interferogram of real data experiment. 


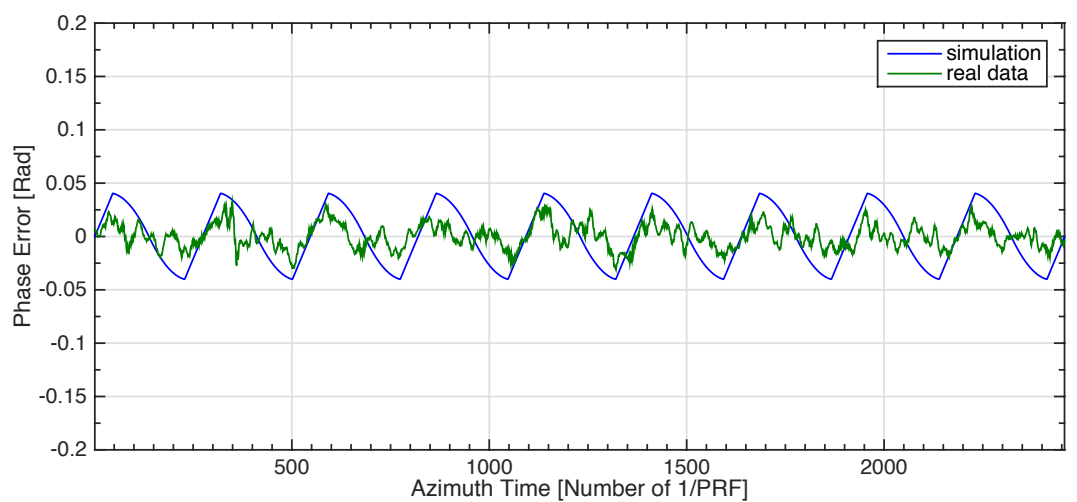

(a)

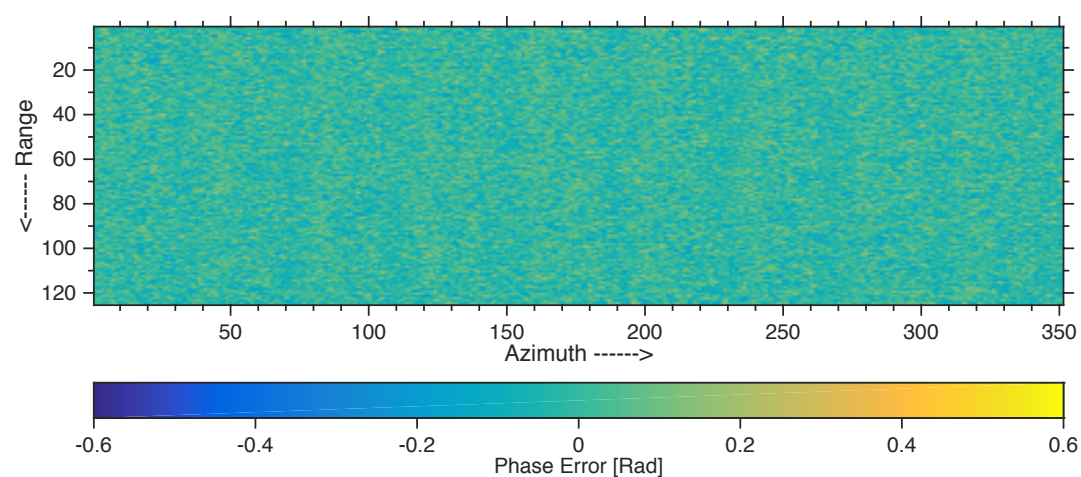

(b)

Fig. 8. Interferometric phase error caused by misregistration. Azimuth spectrum weighted by azimuth antenna pattern and a Kaiser window with a beta value of 2.5. Doppler centroid frequency is $0 \mathrm{~Hz}$. Burst synchronization is $100 \%$. (a) Comparison of simulation and real data results. (b) Interferogram of real data experiment.

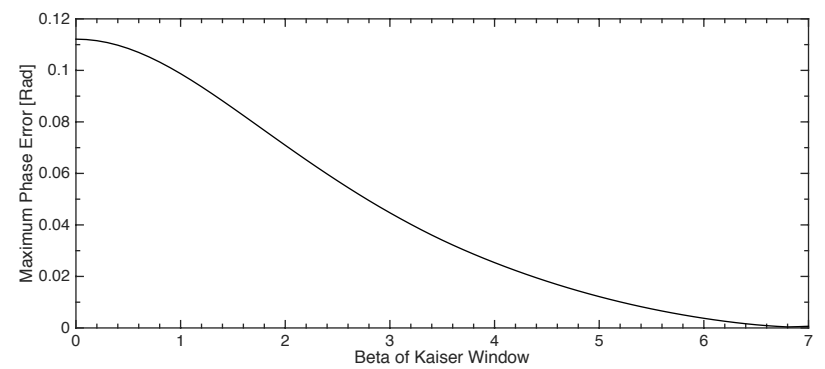

Fig. 9. The simulated relationship of maximum interferometric phase error and the beta value of the Kaiser window applied to the azimuth spectrum. 


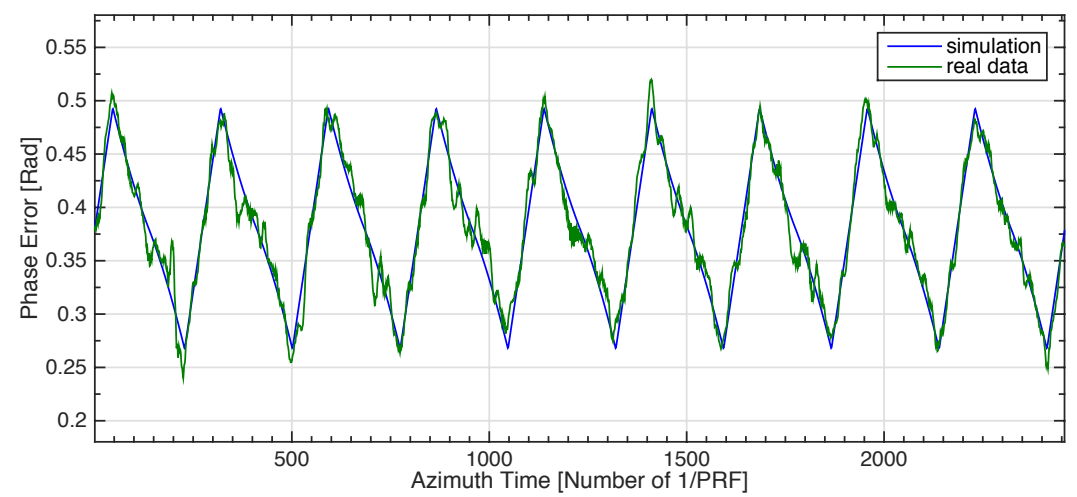

(a)

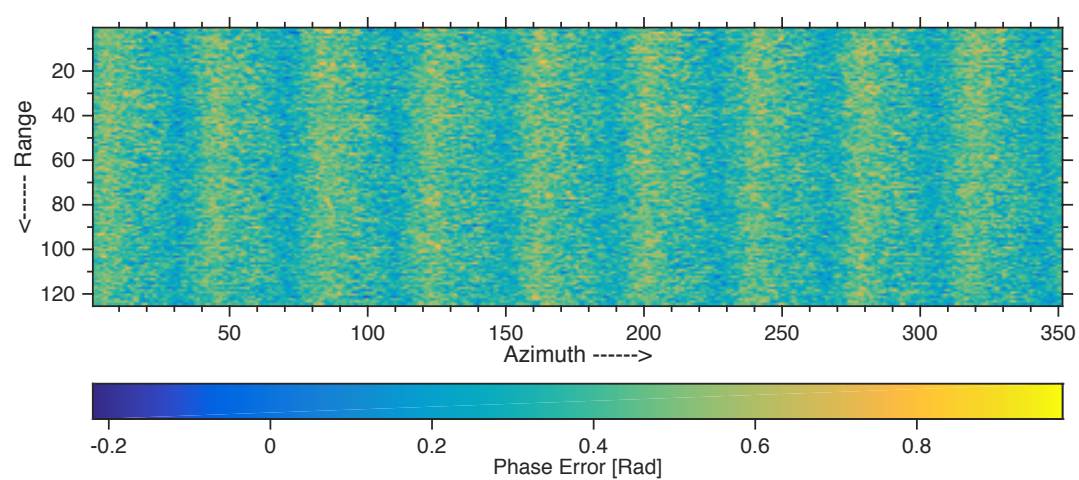

(b)

Fig. 10. Interferometric phase error caused by misregistration. No weighting of azimuth spectrum. Doppler centroid frequency is $200 \mathrm{~Hz}$. Burst synchronization is $100 \%$. (a) Comparison of simulation and real data results. (b) Interferogram of real data experiment.

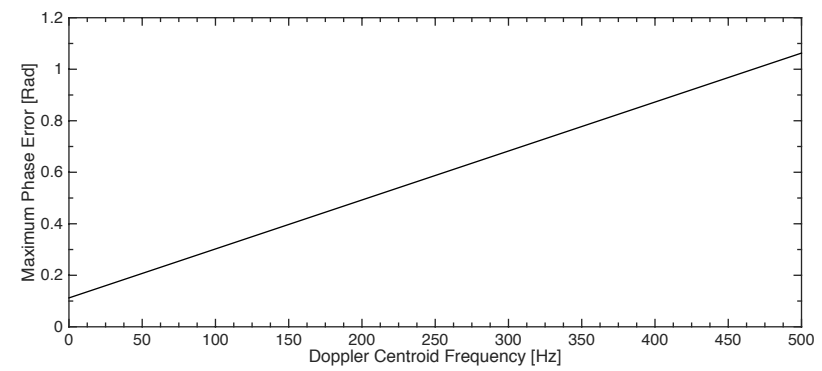

Fig. 11. The simulated relationship of maximum interferometric phase error and the Doppler centroid frequency. 


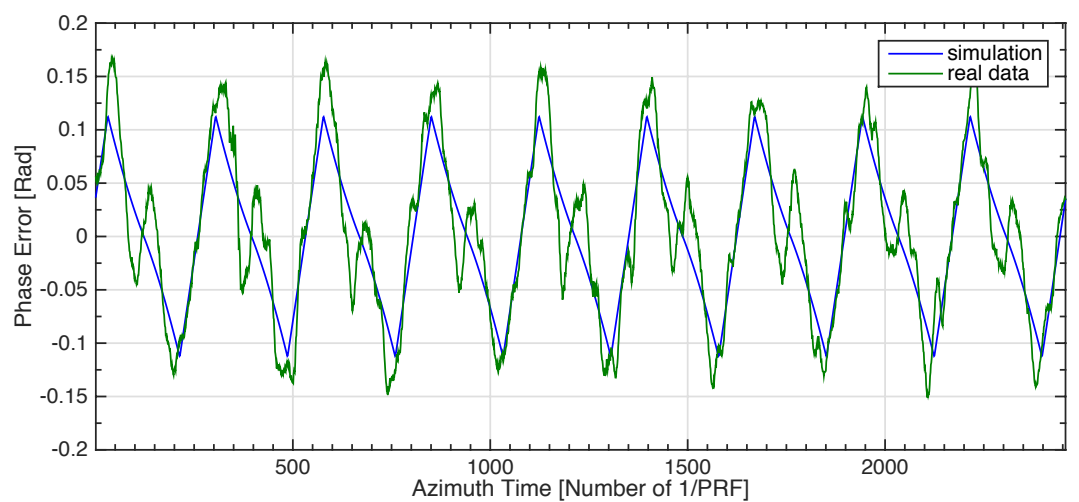

(a)

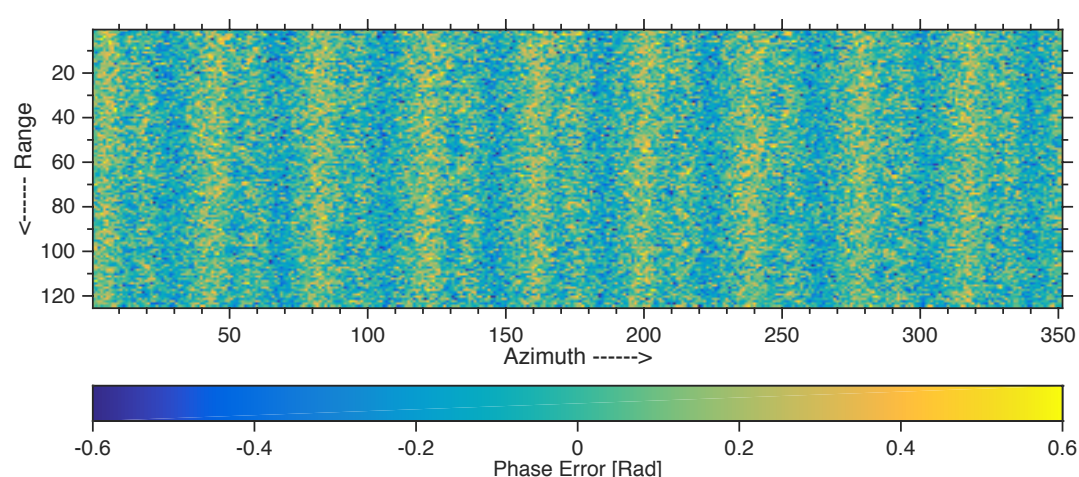

(b)

Fig. 12. Interferometric phase error caused by misregistration. No weighting of azimuth spectrum. Doppler centroid frequency is $0 \mathrm{~Hz}$. Burst synchronization is $67 \%$. (a) Comparison of simulation and real data results. (b) Interferogram of real data experiment. 


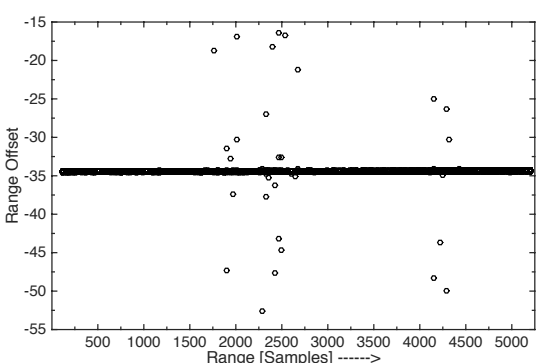

(a)

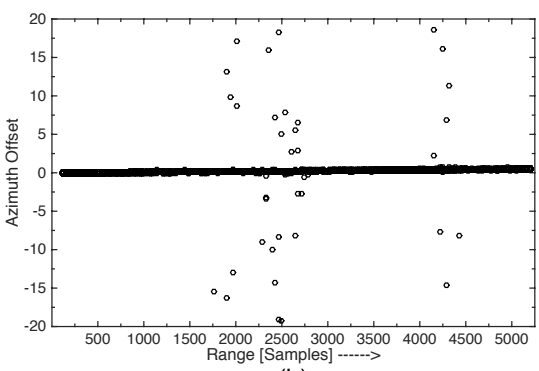

(b)

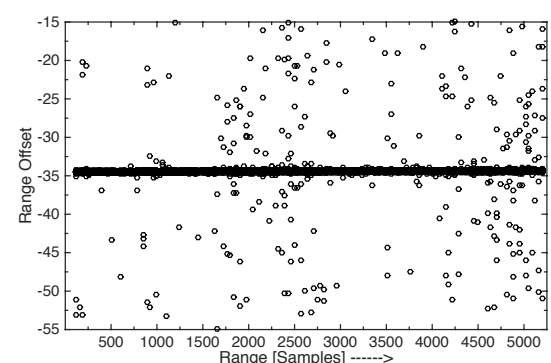

(c)

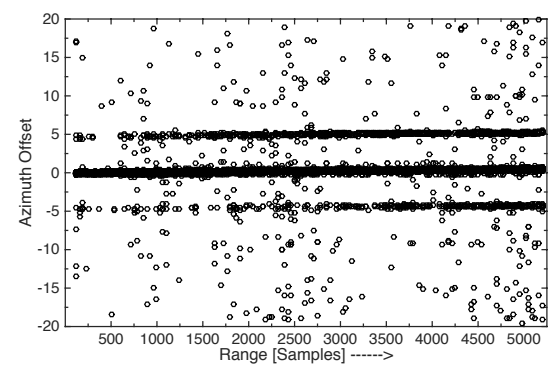

(d)

Fig. 13. Coregistration measurement results. (a) and (b) are the range and azimuth offsets from cross-correlation of stripmap InSAR data, respectively. (c) and (d) are the range and azimuth offsets from cross-correlation of ScanSAR InSAR data, respectively.

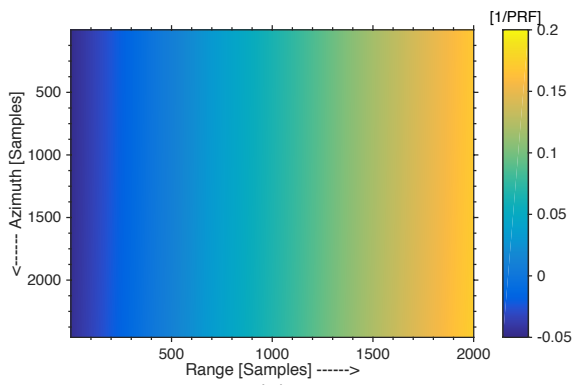

(a)

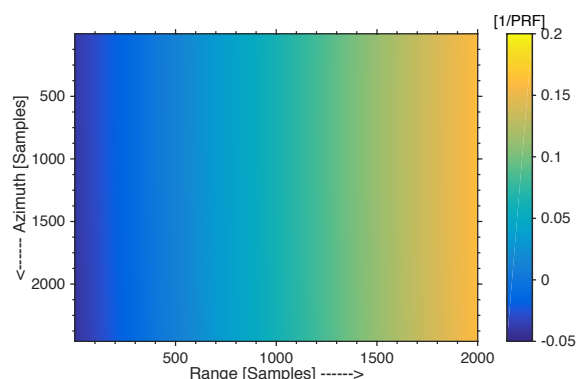

(b)

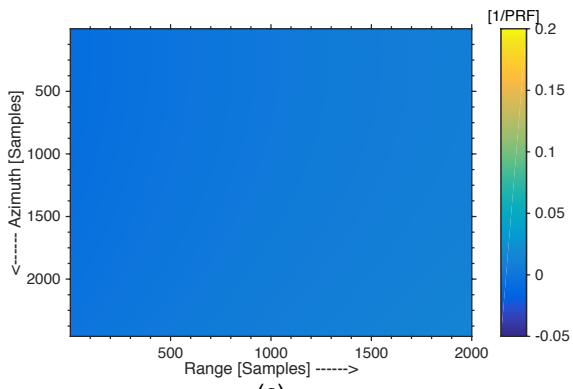

(c)

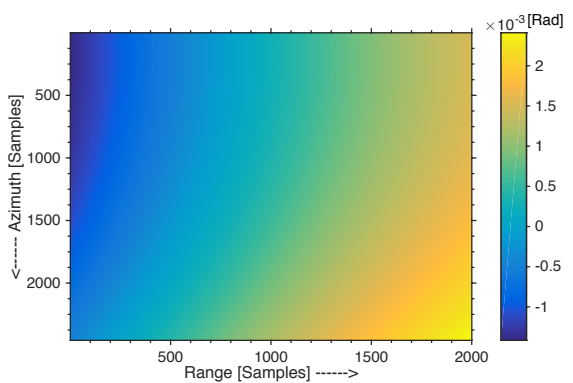

(d)

Fig. 14. (a) Azimuth offset map of stripmap coregistration. (b) Azimuth offset map of ScanSAR coregistration. (c) Difference map of the two azimuth offset maps. (d) ScanSAR interferometric phase error corresponding to the difference map. 

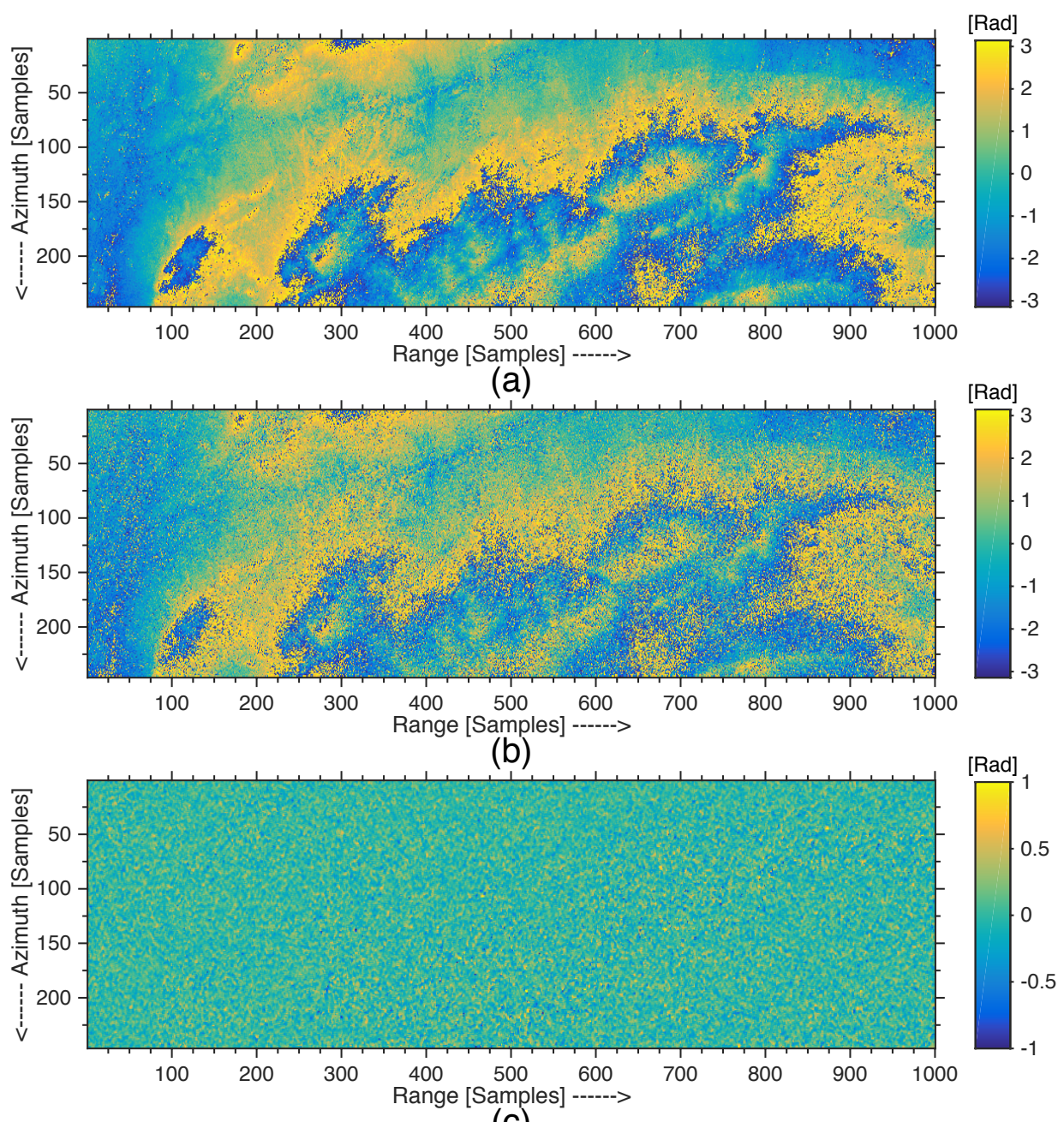

(c)

Fig. 15. (a) Stripmap interferogram. (b) ScanSAR interferogram. (c) Difference of the two interferograms. 


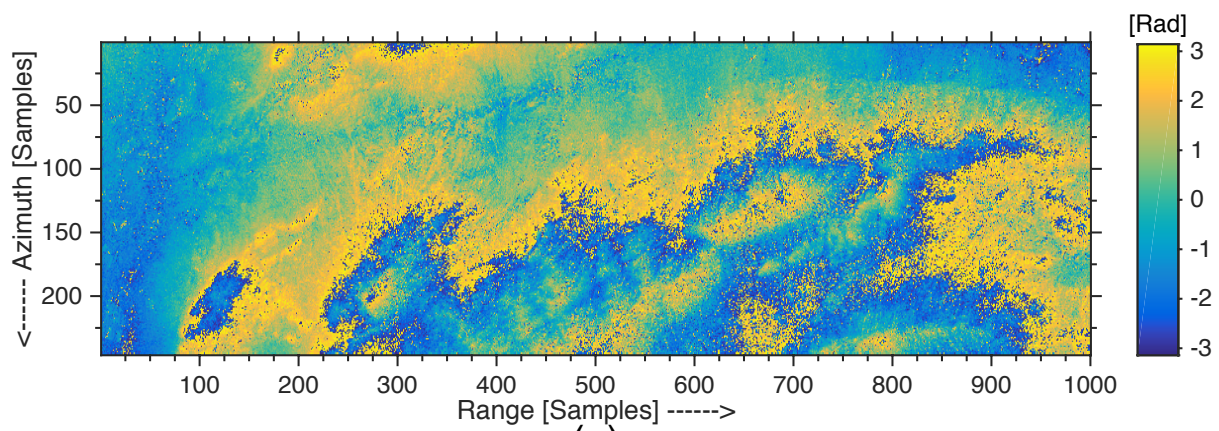

(a)

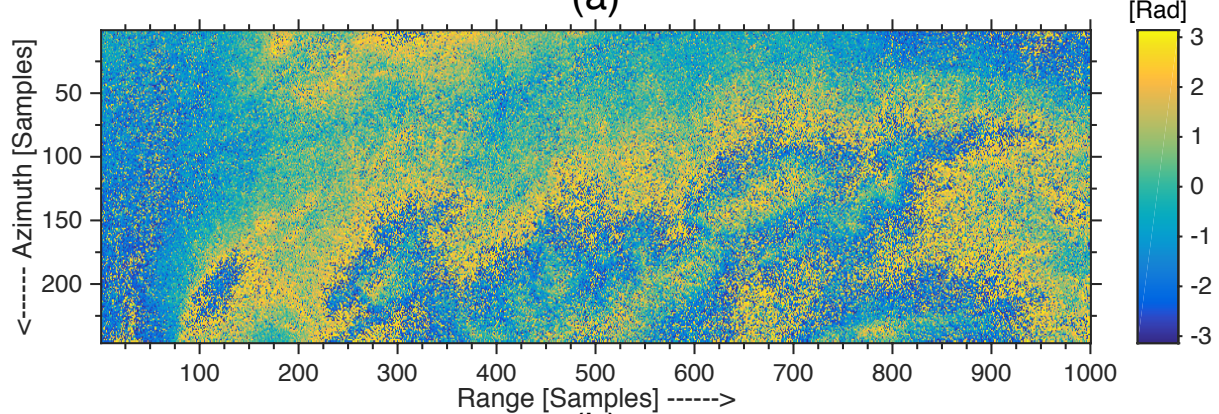

(b)

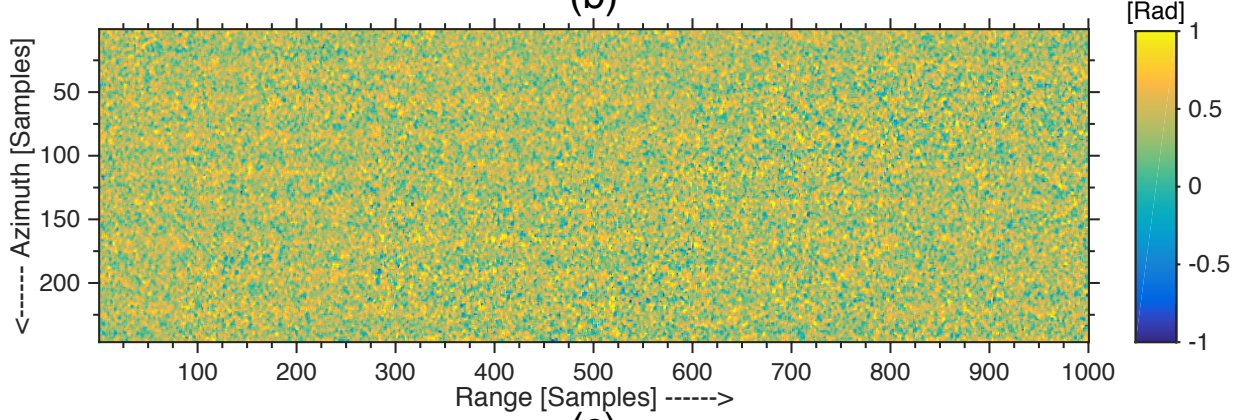

(c)

Fig. 16. (a) Stripmap interferogram. (b) ScanSAR interferogram with a coregistration error of $0.51 / \mathrm{PRF}$ added to azimuth coregistration result. (c) Difference of the two interferograms. 


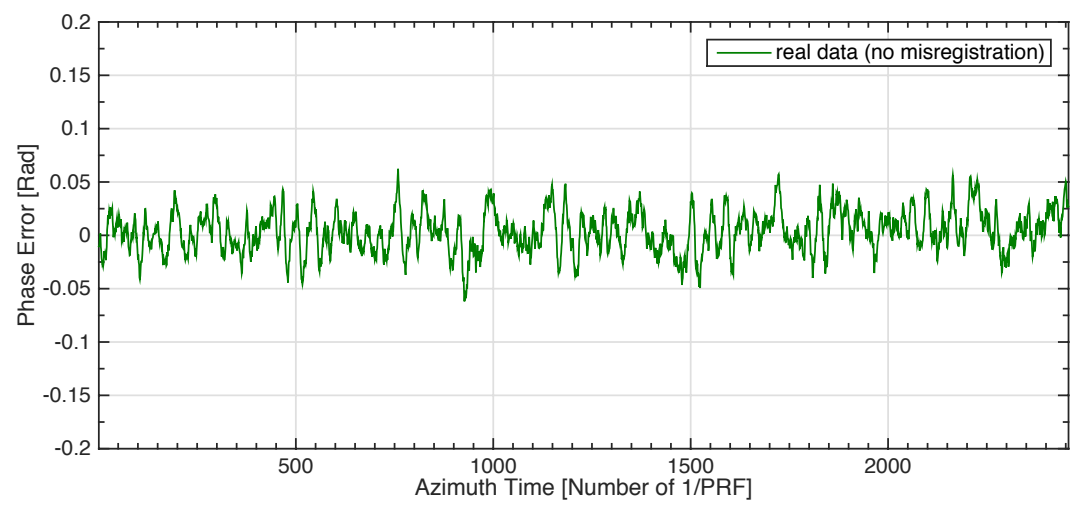

(a)

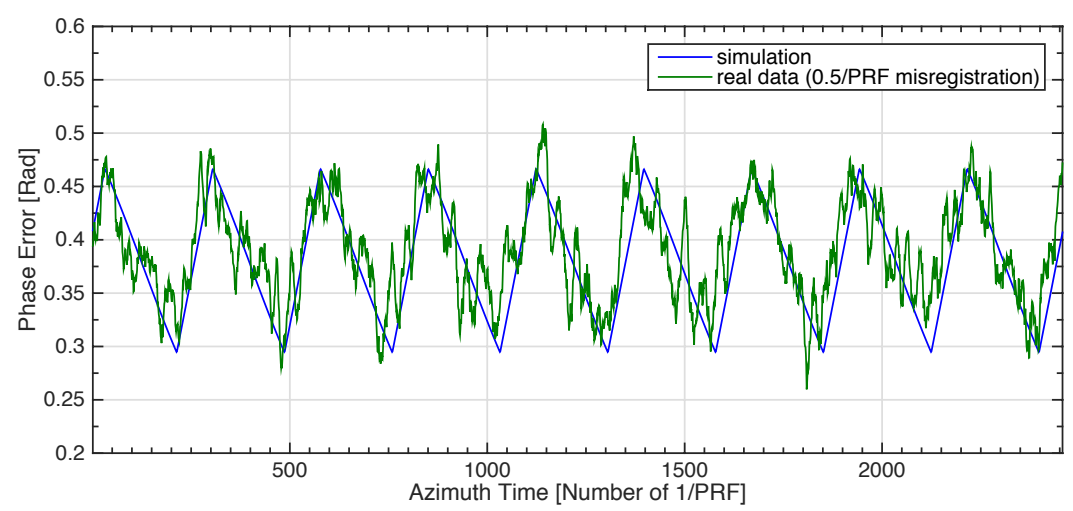

(b)

Fig. 17. Differential interferogram phase after averaging in the range direction. (a) Without the $0.51 / \mathrm{PRF}$ coregistration error. (b) With the $0.51 / \mathrm{PRF}$ coregistration error. 


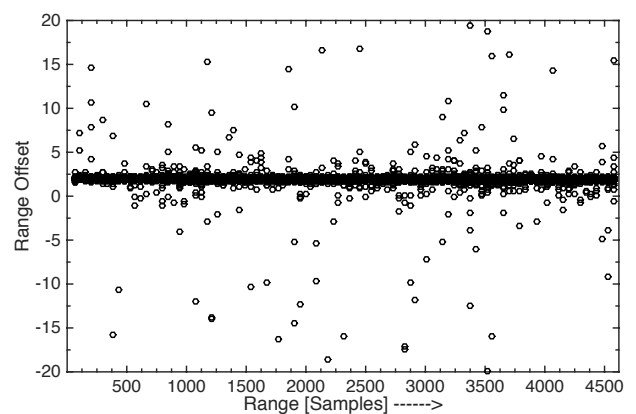

(a)

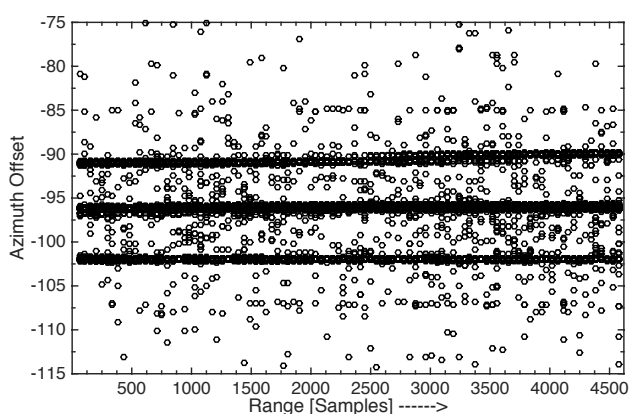

(b)

Fig. 18. Coregistration results of ALOS-2 full-aperture ScanSAR products from two different dates. (a) Range offsets. (b) Azimuth offsets.

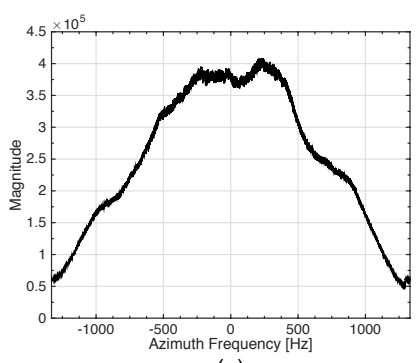

(a)

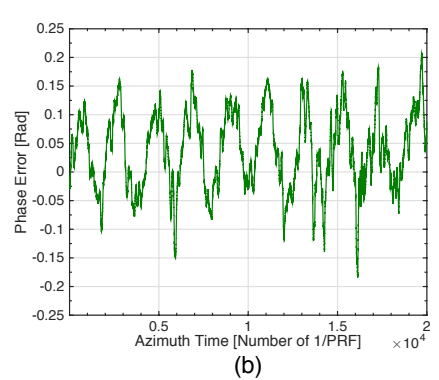

(b)

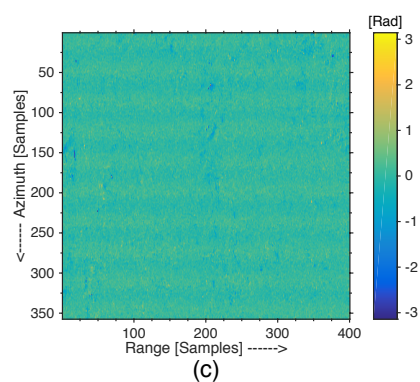

Fig. 19. Subswath 1 of ALOS-2 full-aperture ScanSAR product. (a) Azimuth spectrum. (b) Phase error caused by $11 / \mathrm{PRF}$ coregistration error. (c) Interferogram with 1 1/PRF coregistration error. 
Table I Parameters Used for Simulation

\begin{tabular}{ll}
\hline Parameter & Value \\
\hline PRF [Hz] & 1652.42 \\
Azimuth FM rate [Hz/s] & 2159.04 \\
Total Azimuth Bandwidth Processed [Hz] & 1189.00 \\
Number of Looks & 3 \\
Number of Subswaths & 3 \\
Burst [Number of 1/PRF] & 91 \\
Burst Gap [Number of 1/PRF] & 182 \\
\hline
\end{tabular}

